University of Rhode Island

DigitalCommons@URI

Mechanical, Industrial \& Systems Engineering

Faculty Publications

Mechanical, Industrial \& Systems Engineering

$12-29-2020$

Origins of pressure-induced enhancement in thermal conductivity of hybrid inorganic-organic perovskites

Ashutosh Giri

University of Rhode Island, ashgiri@uri.edu

Follow this and additional works at: https://digitalcommons.uri.edu/mcise_facpubs

The University of Rhode Island Faculty have made this article openly available.

Please let us know how Open Access to this research benefits you.

This is a pre-publication author manuscript of the final, published article.

Terms of Use

This article is made available under the terms and conditions applicable towards Open Access

Policy Articles, as set forth in our Terms of Use.

Citation/Publisher Attribution

Giri A. Origins of pressure-induced enhancement in thermal conductivity of hybrid inorganic-organic perovskites. Nanoscale. 2021 Jan 21;13(2):685-691. doi: 10.1039/d0nr08776a. PMID: 33411864.

This Article is brought to you for free and open access by the Mechanical, Industrial \& Systems Engineering at DigitalCommons@URI. It has been accepted for inclusion in Mechanical, Industrial \& Systems Engineering Faculty Publications by an authorized administrator of DigitalCommons@URI. For more information, please contact digitalcommons-group@uri.edu. 


\title{
Origins of pressure-induced enhancement in thermal conductivity of hybrid inorganic-organic perovskites ${ }^{\dagger}$
}

\author{
Ashutosh Giri, ${ }^{* a}$
}

Received Date

Accepted Date

DOI:00.0000/xxxxxxxxxx

\begin{abstract}
Responses of materials to high pressures unveil important insight into their structure-property relationships that are otherwise masked under ambient conditions. Here, using atomistic simulations, it is shown that the thermal conductivity of methylammonium lead iodide can be drastically increased by more than an order of magnitude with applications of $\sim 60 \mathrm{GPa}$ hydrostatic pressures. The insights gained from the molecular dynamics simulations suggest that although the inorganic constituents contribute significantly to heat flow at ambient pressure, the organic constituents are largely responsible for the drastic increase in thermal conductivity at higher pressures. The application of hydrostatic pressure leads to severe octahedral distortions resulting in higher degrees of confinement of the organic molecules and the concomitant hardening of their vibrational density of states to high frequencies encompassing a very broad spectral range that are otherwise unavailable at ambient pressure. This is shown to drastically improve the overall thermal conductivity even though the contributions from the inorganic constituents remain largely unchanged throughout the pressure range studied in this work.
\end{abstract}

Organic-inorganic hybrid perovskites possess remarkable physical properties, positioning them as ideal candidates for use in emerging technologies such as in electrochemical energy storage, thermoelectric applications, $\frac{1-5}{5}$ and primarily in solar cells achieving efficiencies $>20 \%$. 6 - For example, the long carrier lifetimes and strong band-edge absorption in hybrid perovskites motivate their use in planar photovoltaics with enhanced charge transport characteristics, thus increasing the power-conversion efficiency. ${ }^{910}$ Recently, further enhancement in their efficiencies as materials in photovoltaics have been achieved through pressure, where lattice compression allows for the systematic control of the structure without modifying the chemical composition. ${ }^{11 \mid 12}$ By compressing the lattice in a controllable manner, hydrostatic pressure has been linked with bandgap narrowing and carrier-lifetime prolongation, 13 14 enhancement in photoluminescence intensity. 15 The structural, optical and electronic properties of hybrid perovskites have been shown to be fully retainable after pressure release, demonstrating their potential for applications involving transient changes in their physical properties. 16 Moreover, pressure studies have also shed light into

\footnotetext{
${ }^{a}$ Department of Mechanical, Industrial and Systems Engineering, University of Rhode Island, Kingston, RI 02881, USA ; E-mail:ashgiri@uri.edu

$\dagger$ Electronic Supplementary Information (ESI) available: [details of any supplementary information available should be included here]. See DOI: 00.0000/00000000. \$ Additional footnotes to the title and authors can be included e.g. 'Present address:' or 'These authors contributed equally to this work' as above using the symbols: $\downarrow$, §, and ๆ. Please place the appropriate symbol next to the author's name and include a $\backslash$ footnotetext entry in the the correct place in the list.
}

important structure-property relationships of hybrid perovskites such as demonstrating semiconductor to metal transition at pressures above $60 \mathrm{GPa} .1117$ However, the understanding of thermal transport properties of metal halide perovskites at high pressures has been unexplored in prior literature. Since the thermal properties are central to the further advancements of these materials for use as next-generation photovoltaic and semiconductor systems, a systematic study emphasizing the dependence of thermal properties on pressure is quintessential to fully realize their potential in various types of energy related technologies.

The thermal conductivity of organic-inorganic hybrid perovskites at ambient pressure has been investigated both exper-

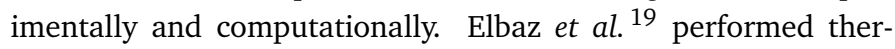
moreflectance measurements to report thermal conductivities in the range of 0.34 to $0.73 \mathrm{~W} \mathrm{~m}^{-1} \mathrm{~K}^{-1}$ for various 3D single crystal lead halide perovskites. They ascribed the difference between the various hybrid perovskites to be dictated by the varying phonon group velocities. Similarly, molecular dynamics simulations have been conducted on the prototypical methylammonium lead iodide, $\mathrm{CH}_{3} \mathrm{NH}_{3} \mathrm{PbI}_{3}\left(\mathrm{MAPbI}_{3}\right)$, to show that the low group velocities of acoustic phonons and strong phonon-phonon scattering mechanisms are the main factors responsible for the ultralow thermal conductivities in these crystalline materials. $\frac{20 \mid 21}{2}$ Indeed, the role of the dynamic disorder created by the motion of the organic molecules and their coupling with the inorganic lattice has been linked to the reduced overall thermal conductivities in these materials. 22 Although the aforementioned works shed 
(a)

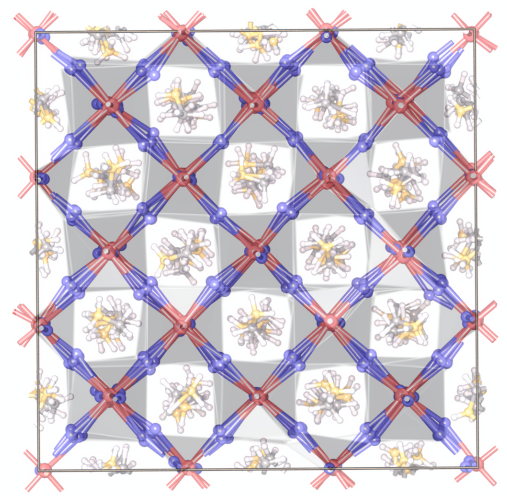

(b)

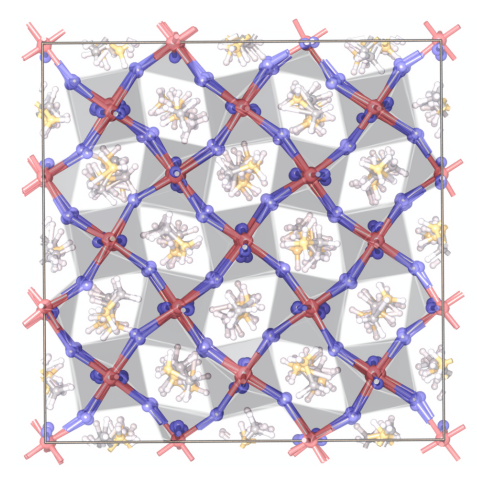

(c)

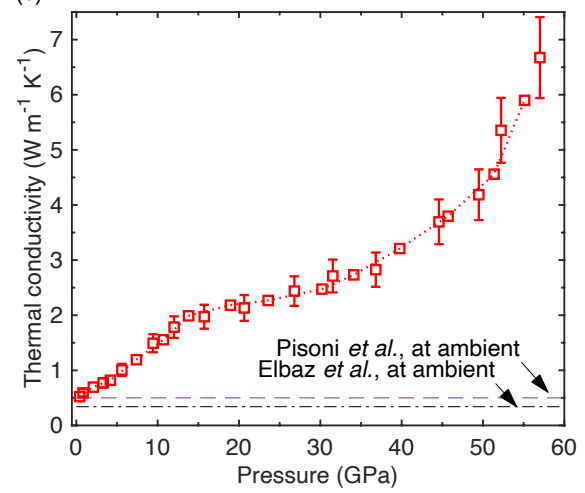

Fig. 1 Snapshots of the computational domains showing the structures of $\mathrm{MAPbl}_{3}$ at (a) low $(0.4 \mathrm{GPa})$ and (b) high (7.4 GPa) pressures. The application of hydrostatic pressures is shown to distort the inorganic octahedra. (c) Equilibrium molecular dynamics-predicted thermal conductivity of MAPbl ${ }_{3}$ as a function of hydrostatic pressure. The application of hydrostatic pressures is shown to have a non-monotonic increase in thermal conductivity by more than $\sim 10 \times$ at pressures of $\sim 55 \mathrm{GPa}$.

light on some important heat transfer mechanisms in these novel materials, the following fundamental questions still remain unanswered:

- What effect does pressure have on the thermal conductivity of hybrid perovskites?

- What are the separate contributions from the organic and inorganic constituents to the total thermal conductivity and how do these contributions change with the application of hydrostatic pressure?

This work seeks to answer these questions via atomistic simulations.

In general, the application of hydrostatic pressures have been shown to reveal important insights into properties of condensed matter that are otherwise not descernible under ambient pressure. For example, high pressure studies have shown the ability to modulate the thermal conductivity of various materials over different phases, 26-30 decrease interfacial thermal resistance, $\underline{3132}$ and tune the strength of scattering between electrons and phonons in metals, 33 thereby advancing our understanding of the microscopic processes occurring in condensed matter. Therefore, in this work, the application of hydrostatic pressure is used to study the thermal response of the prototypical hybrid perovskite $\left(\mathrm{MAPbI}_{3}\right)$. The results from the molecular dynamics simulations show that hydrostatic pressure induces a nonmonotonic increase in thermal conductivity, which is dominated by the contributions from the organic constituents at high pressures. At ambient conditions, it is found that the inorganic constituents contribute to $\sim 50 \%$ of the total thermal conductivity (inline with the prior findings showing the significant role of the inorganic lattice on the thermal properties of hybrid perovskites at ambient pressure). However, as the pressure is increased, the increased octahedral tilting reduces the contributions from the inorganic constituents even though pressure leads to lattice stiffening, which generally results in an increase in the thermal conductivity of solids. The broadening of the vibrational density of states (in a broad spectral region) of the organic constituents drastically increases the thermal conductivity at higher pressures and leads to more than an order of magnitude higher thermal conductivity at pressures of $\sim 60 \mathrm{GPa}$ as compared to that at ambient pressure. These insights gained from the high pressure study have the potential to increase the scope of hybrid metal halide perovskites for energy-based applications.

As the choice of the interatomic potential is crucial in predicting the correct vibrational physics, the $a b$ initio-based potential developed by Mattoni et al., ${ }^{35}$ which has been used previously to study the thermal properties of $\mathrm{MAPbI}_{3}$ under ambient pressure is implemented in this work. $\frac{2124}{2}$ The potential was specifically developed to correctly reproduce the total energy of the hybrid perovskite under hydrostatic deformations and replicate the energy profile as the molecular constituents reorient themselves with respect to the deforming inorganic lattice. Therefore, the choice of the potential is appropriate to describe the changes in the thermal characteristics with the application of hydrostatic pressure. Figure 1 shows examples of the computational domains at low (0.4 $\mathrm{GPa})$ and relatively higher $(7.4 \mathrm{GPa})$ pressures to highlight the inorganic octahedral tilts that result from higher pressures, which has major implications on the overall heat transfer of $\mathrm{MAPbI}_{3}$ as will be discussed in more detail below.

The thermal conductivities of the $\mathrm{MAPbI}_{3}$ computational domains are calculated with varying hydrostatic pressures of up to $\sim 60 \mathrm{GPa}$ from equilibrium molecular dynamics (EMD) simulations (see Supplementary for further details of the computational domain setup and thermal conductivity predictions including the calculation of the heat flux autocorrelation function and the time integral for the equilibrium calculations). Figure 1 1 s shows the thermal conductivity of $\mathrm{MAPbI}_{3}$ as a function of hydrostatic pressure predicted from our atomistic simulations. At ambient pressure, the predictions from the EMD simulations agree with the experimental measurements in Refs. 19 and 23 (as shown by the dashed lines). However, it is cautioned that there could be size effects due to neglecting very long-wavelength phonon modes as there are limitations with the computational domain sizes for 
(a)

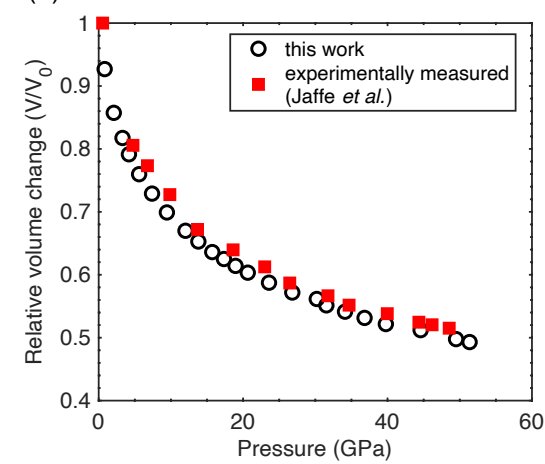

(c)

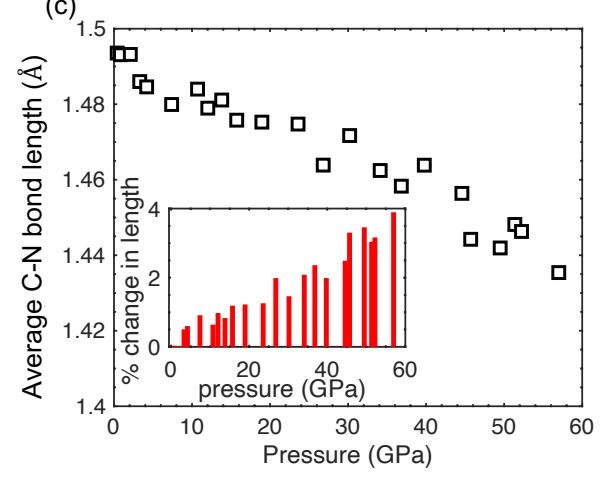

(b)

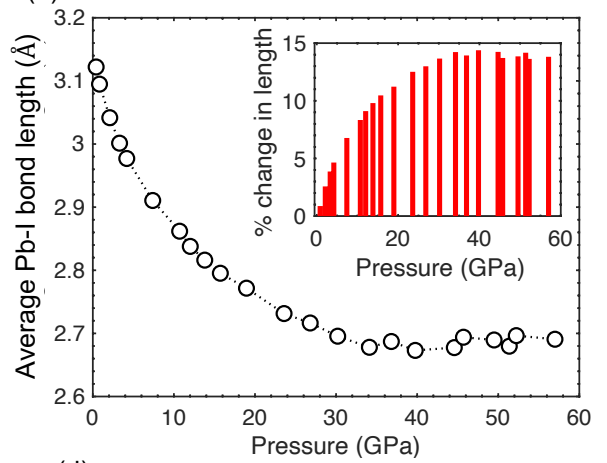

(d)

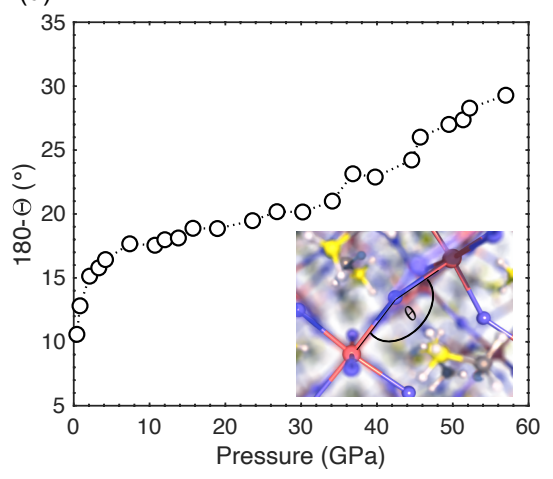

Fig. 2 (a) The relative change of volume predicted via molecular dynamics simulations as a function of applied pressure for MAPbl 3 . For comparison, the experimental results from Ref. 17 are also included. Average change in the bond length as a function of pressure for (b) Pb-I and (c) C-N. While the change in the $\mathrm{Pb}-\mathrm{l}$ bond length mimics the positive curvature of the volume change, the $\mathrm{C}-\mathrm{N}$ bond length change is less pronounced in comparison and shows a more monotonic decrease. The insets show the percentage change in the bond lengths with increasing pressures. (d) The relative change in the $\mathrm{Pb}-\mathrm{I}-\mathrm{Pb}$ bond angle from a perfect $180^{\circ}$ (as depicted in the inset) as a function of applied pressure.

predicting the thermal conductivity with the Green-Kubo (or the EMD) approach. Therefore, although the convergence of thermal conductivity with system size (as shown in Fig. S6 of the Supplemental) does provide confidence in these predictions, it is noted that these thermal conductivity predictions should be taken as lower bounds.

As shown in Fig. 17, the application of pressure is shown to have a more significant increase in thermal conductivity at lower pressures and for pressures $>40 \mathrm{GPa}$. The overall thermal conductivity of $\mathrm{MAPbI}_{3}$ increases by more than 10 fold at pressures of $\sim 58 \mathrm{GPa}$. In what follows, the structural origins of this "nonmonotonic" increase is investigated with additional analysis and simulations to reveal the microscopic mechanisms that control the enhanced thermal properties with hydrostatic pressure.

To gain further confidence in the thermal conductivity predictions and to provide additional support for the choice of the interatomic potential, we compare the relative change in volume with pressure predicted via the MD simulations with the experimentally measured changes in volume that have been conducted under pressures of up to $\sim 50 \mathrm{GPa}$ in Fig. 2]. 18 The agreement between the results from the experiments and the predictions from the simulations suggests that the interatomic potential can correctly reproduce the equation of state for $\mathrm{MAPbI}_{3}$. Moreover, the positive curvature of the relative volume changes as shown in Fig. 2 a suggests that the bonds stiffen, which consequently leads to the hardening of the phonon modes as will be discussed in more detail below.

From a structural perspective and to relax the inorganic lattice, the application of hydrostatic pressure causes octahedral tilting (see Fig. S1 of Supplemental and Fig. 1) and length contraction of the $\mathrm{Pb}-\mathrm{I}$ bonds to occur (by as much as $\sim 15 \%$ at pressures $>40 \mathrm{GPa}$ as shown in Fig. 2p). However, the bond lengths in the organic constituents are relatively unaffected (as shown in Fig. 2f) with the average $\mathrm{C}-\mathrm{N}$ bond lengths only changing by $\sim 4 \%$ with pressures of up to $60 \mathrm{GPa}$. Furthermore, the positive curvature in the volume change is qualitatively replicated in the bond length changes for the Pb-I bonds, whereas, the change is more monotonic for the $\mathrm{C}-\mathrm{N}$ bond suggesting that the hydrostatic pressure mainly affects the inorganic framework, which concomitantly will have an influence on the microscopic dynamics of the organic constituents surrounded by the $\mathrm{PbI}_{6}$ octahedra. Therefore, to get a more quantitative measure of the octahedral tilting with pressure, the deviations in the average $\mathrm{Pb}-\mathrm{I}-\mathrm{Pb}$ bond angles as illustrated in the inset of Fig. $2 \mathrm{~d}$ is calculated. As is clear from Fig. $2 \mathrm{~d}$, the change in the average $\mathrm{Pb}-\mathrm{I}-\mathrm{Pb}$ angle from a perfect $180^{\circ}$ increases with increasing pressure. However, the bond angle change exhibits a more significant increase at presses $<10$ $\mathrm{GPa}$ and pressures $>40 \mathrm{GPa}$. This "non-monotonic" deviation is qualitatively similar to the increasing trends in thermal conductivity with pressure as shown in Fig. 1k, which suggests that the 
(a)

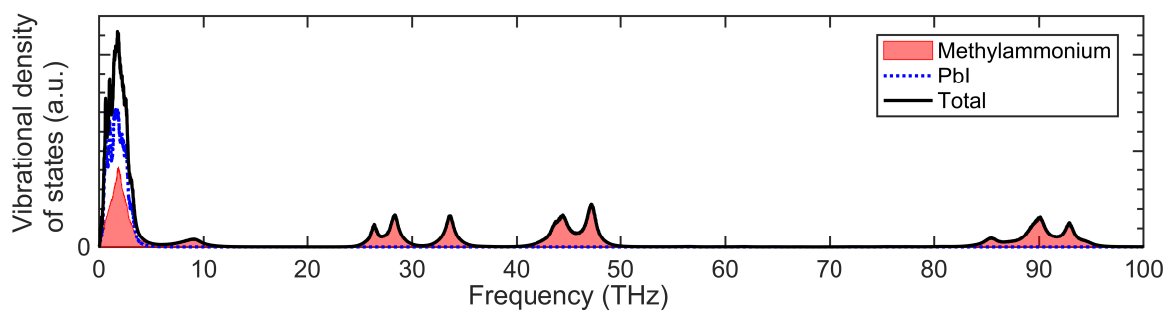

(b)

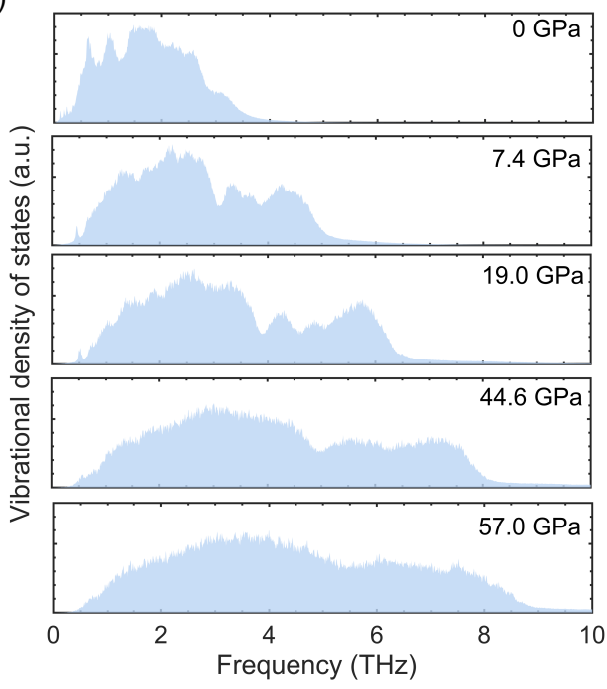

(c)

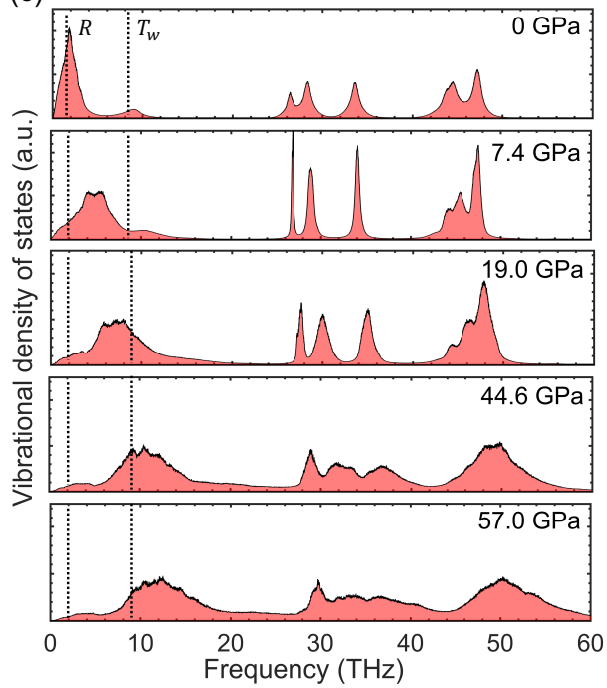

Fig. 3 Vibrational density of states computed from the Fourier transform of the autocorrelation of the velocities of the atoms in the MAPbl 3 computational domain. (a) The separate contributions from the organic and inorganic constituents along with the total vibrational density of states for MAPbl${ }_{3}$ at ambient pressure is shown. Vibrational density of states of the (b) inorganic and (c) organic constituents at different pressures showing the hardening of the vibrations in both constituents as the pressure is increased. The modes represented by $R$ and $T_{w}$ for the organic cations are prescribed to rotational modes and twisting modes around the $\mathrm{C}-\mathrm{N}$ axis, respecively. 36

intra- and inter-octahedral distortions can significantly influence the overall thermal transport in these hybrid perovskites. These distortions also affect the interaction energy between the cations and the inorganic framework as quantitatively demonstrated in Fig. S10b of the Supplemental where the interaction energy (not including the long-range Coloumbic interactions) remains constant till $\sim 10$ GPa hydrostatic pressure and increases monotonically for higher pressures. For hydrostatic pressures $>40 \mathrm{GPa}$, the pair-wise interaction energy becomes positive due to the considerable repulsion at short distances between the cations and the inorganic cage.

Next, the effect of pressure on the vibrational density of states (DOS) of $\mathrm{MAPb}_{3}$ is investigated (the details of the calculations are given in the Supplemental). Figure 33 shows the separate contributions to the total DOS from the organic and inorganic constituents. Note, the calculated total DOS is qualitatively similar to that calculated via density functional theory calculations by Brivio et al. and reproduces all the main features of the total DOS. ${ }^{37}$ More specifically, the low frequency band in the 0 to 7 $\mathrm{THz}$ frequency range, which mainly originates from the motion of the inorganic octahedrons, and the peak at $\sim 9 \mathrm{THz}$, which corresponds to the twisting motion of the methylammonium cation around the C-N bond is reproduced in the MD simulations. 20
The higher energy vibrations that are entirely due to the organic molecules (and related to bond bending, stretching and dihedral rotations) are a direct consequence of the very stiff $\mathrm{C}-\mathrm{N}, \mathrm{C}-\mathrm{H}$ and $\mathrm{N}-\mathrm{H}$ bonds. As the pressure is increased, the vibrational landscape of the inorganic as well as the organic constituents change drastically as shown in Fig. 33 and 3k, respectively. The increase in the spring constant, as quantitatively demonstrated by the increasing interaction force between $\mathrm{Pb}$ and I atoms and the increasing interaction energy between the cation and the inorganic framework with increasing pressure (as shown in Supplemental Fig. S10), leads to the hardening of the phonon modes in both the inorganic and organic constituents, respectively. However, the low frequency peak of the organic constituent shifts to higher frequencies more so than the peak of the inorganic constituent (which is also considerably broadened to frequencies of up to $9 \mathrm{THz}$ at higher pressures). In contrast to the inorganic framework's response to pressure, the DOS for the organic constituents hardens to higher frequencies mainly due to modified interactions with the halogens due to the shrinking of the voids in the inorganic framework, which ultimately leads to the broadening of the vibrations that reaches the intermediate frequencies in the 25 to $60 \mathrm{THz}$ range (that were previously isolated at ambient pressure; see Figs. 3 and 3 F). Moreover, the DOS at $\sim 1.9 \mathrm{THz}$ that are 
(a)

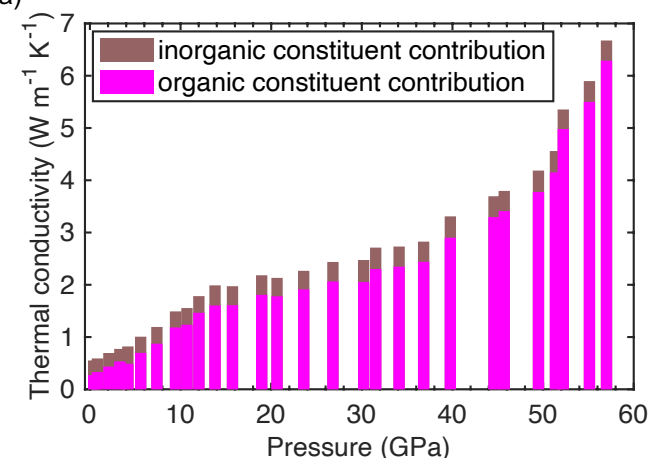

(b)

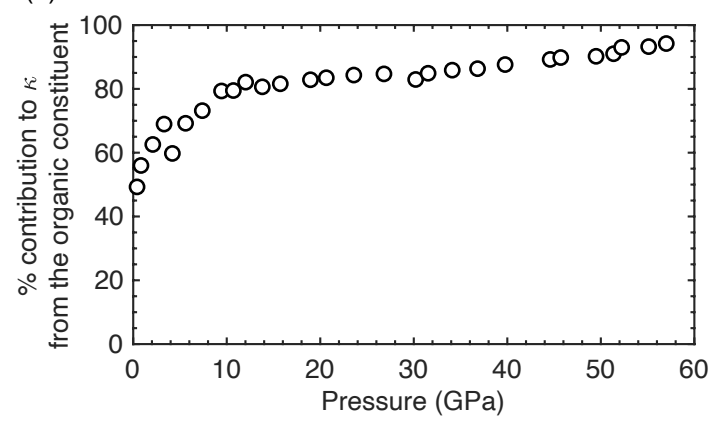

(c)
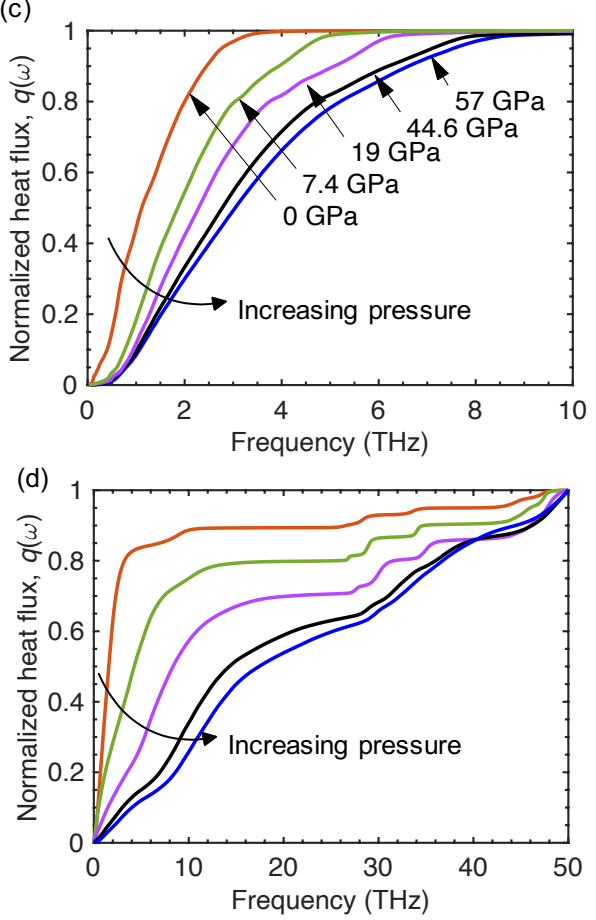

Fig. 4 (a) Separate contributions to the total thermal conductivity from the organic (pink) and inorganic (brown) constituents as a function of pressure. (b) The precent contribution from the organic constituents to the total thermal conductivity as a function of pressure. At ambient pressure, the inorganic constituent has significant contributions to the heat flow, whereas, at higher pressures, the organic constituent dominates thermal transport. Normalized spectral heat flux calculations for (c) the inorganic and (d) the organic constituents at different hydrostatic pressures.

largely prescribed to the rotational motion of the organic cations (as represented by $R$ in Fig. 35) diminish gradually with the application of hydrostatic pressure, while the DOS for the modes at $\sim 9$ $\mathrm{THz}$ that are prescribed to the twisting of motion around the $\mathrm{C}-\mathrm{N}$ axis $\left(T_{w}\right)$ are increased with increasing pressure. $\frac{36}{36}$ As it will be shown next, this blue-shift and extended broadening in the DOS of the methylammonium cation leads to the drastically enhanced thermal conductivities in $\mathrm{MAPbI}_{3}$ at high hydrostatic pressures.

The separate contributions from the organic and inorganic constituents to the total thermal conductivity (details in Supplemental) is shown in Fig. 4. Whereas the contributions from the inorganic part are relatively constant throughout the entire pressure range studied in this work as represented in the bar graph by the darker shade in Fig. 4 , the contributions from the organic molecules to the overall thermal conductivity dramatically increases with pressure as represented by the lighter (pink) shade. At ambient pressure, the inorganic framework has significant contribution to the total thermal conductivity with the organic counterparts contributing to $\sim 50 \%$ as shown in Fig. 4 p. Their contributions steadily increase to $\sim 80 \%$ with pressures of up to 10 GPa and eventually at very high pressures, almost all of the heat is carried by the organic constituents. The limited contribution from the inorganic framework can potentially be attributed to the severe distortions of the $\mathrm{PbI}_{6}$ octahedra with pressures as quantitatively demonstrated by the change in the $\mathrm{Pb}-\mathrm{I}-\mathrm{Pb}$ bond angles with pressure as shown in Fig. $2 \mathrm{~d}$ and schematically illustrated for different pressure cases in Fig. S1 of the Supplemental. However, the coupled interaction between the framework and the organic molecules (as exemplified by the similarities in the qualitative trends in $\mathrm{Pb}-\mathrm{I}-\mathrm{Pb}$ bond angle changes and thermal conductivity increase with pressure as mentioned above), limits the dynamic motion of the molecules, which is evident from the decrease in their mean square displacement (see Fig. S9 of Supplemental) inside the deformed inorganic framework; the dynamics and the normal frequencies of the methyl ammonium cations are affected by the electrostatic potential of the inorganic framework. 36 The modification of the cation dynamics due to pressure is further evident from the calculated trajectories of the organic cations inside the reduced volume of the inorganic cages with the increasing hydrostatic pressures (see Supplemental Fig. S11). As pressure is increased, the trajectories of the organic cations are greatly reduced inside the shrinking inorganic cages. The restricted trajectories along with the increasing interaction energy between the inorganic framework and the organic cations (see Supplemental Fig. S10b) leads to the large broadening of the available vibrational DOS of the organic constituents and concomitantly to the drastic increase in the total thermal conductivity of $\mathrm{MAPbI}_{3}$ with increasing hydrostatic pressure.

As mentioned above, the contributions from the inorganic framework to the total thermal conductivity remains largely unchanged with pressure. This result is a bit surprising given the fact that thermal conductivity usually increases in inorganic crystals with the application of compressive forces due to the lattice stiffening and the vibrational spectrum broadening to higher frequen- 
cies. $\frac{2629}{29}$ To shed more light into this, calculations of the spectrally decomposed heat flux according to the procedure outlined in Refs. 3839 is carried out. As shown in Fig. 4k, the calculations of the normalized spectral heat flux at various pressure conditions for the inorganic constituents suggest that the heat carrying vibrations in the inorganic framework shift to higher frequencies as the hydrostatic pressure is increased. While the contributions from higher frequencies increase with pressure and would lead to an increase in the thermal conductivity (as is the case for most inorganic crystals), the octahedral distortions could potentially lower the lifetimes of the low frequency vibrations and lead to a reduction in thermal conductivity of these modes, thus offering two competing mechanisms for heat conduction. In fact, as shown by the spectral heat flux calculations, the reduction in contribution from the low frequency vibrations $(<2 \mathrm{THz})$ that carry $\sim 80 \%$ of the heat under ambient conditions and the increase in contributions from the relatively higher frequency vibrations lead to an unchanged overall contribution to the total thermal conductivity from the inorganic framework as the pressure is increased.

To understand the contributions of the inter- and intramolecular interactions to the thermal conductivity, additional calculations of the DOS are conducted by treating the MA cations as rigid bodies inside the $\mathrm{PbI}_{6}$ octahedra; as rigid bodies, the DOS of the cations as shown in Fig. S will correspond to vibrations resulting from intermolecular interactions only. These calculations along with the spectrally decomposed heat flux for the organic cations (with the full degrees of freedom included in the calculations as shown in Fig. 4d) point to the fact that intermolecular interactions that are associated with the comparatively low frequency spectrum can contribute substantially to the total thermal conductivity for all pressures considered. It is also apparent from Fig. $4 \mathrm{~d}$ that as pressure is increased, the contributions from higher frequency vibrations increase suggesting a more prominent role of intramolecular vibrations at higher pressures. However, it is noted that Caddeo et al. ${ }^{[24}$ have shown that by freezing the intramolecular degrees of freedom of the MA cations, the thermal conductivity of $\mathrm{MAPbI}_{3}$ is considerably enhanced, which they ascribe to lowered scattering of phonon modes of the inorganic framework. It is also interesting to note that their calculations for the case when rotations of the molecules are ignored (while all the rest of the degrees of freedom are available for the MA cations) show that thermal conductivity is relatively unaffected by the rotational motion. Therefore, it would be interesting to study the effect of various degrees of freedom of the organic cations on the pressure dependent thermal conductivity of $\mathrm{MAPbI}_{3}$, which deserves a comprehensive investigation, but is beyond the scope of the current work.

The motion of the organic cations, whether freely rotating or locked in a specific orientation inside the voids of the inorganic framework has been predominantly linked to their relative size and shape as compared to the void size in hybrid perovskites. $\frac{40}{40}$ As such, the strength and extension of the H-bond network that couples the organic to the inorganic constituents significantly governs the microscopic dynamics of the cations. In this respect, prior works have shown that even though the electronic band structure is mainly dictated by the inorganic framework, the interac- tions of the organic cations with the 12 surrounding halogens affect the framework and the overall electronic and optical properties. 40 42 This study further highlights the important interplay between the organic and inorganic constituents in dictating their physical properties and also sheds light on the tunable and enhanced thermal properties of hybrid perovskites with pressure, which has been unexplored.

In summary, we have shown that hydrostatic pressure can be used as an efficient method to tune the thermal conductivity of hybrid inorganic-organic perovskites. Through atomistic simulations, we have shown that although the inorganic constituents contribute significantly to heat flow at ambient pressure, the organic constituents are largely responsible for the drastic increase in thermal conductivity at higher pressures leading to more than an order of magnitude increase in thermal conductivity with pressures of $\sim 60 \mathrm{GPa}$. The application of hydrostatic pressure leads to severe octahedral distortions resulting in higher degrees of confinement of the organic molecules and the concomitant hardening of their vibrational density of states to high frequencies encompassing a very broad spectral range that are otherwise unavailable at ambient pressure. This is shown to drastically improve the overall thermal conductivity even though the contributions from the inorganic constituents remain largely unchanged throughout the pressure range studied in this work. Lattice compression through hydrostatic pressure, not only creates large responses in their photophysical and charge transport properties, but also leads to drastically higher thermal conductivities in these novel materials. The insights gained from high-pressure research and the pressure-driven thermodynamic changes present an ideal platform to understand the intrinsic structure-property relationship, thus providing guidelines for future and next-generation materials by design for applications in photovoltaics and flexible energy harvesting devices that have the potential to redefine how we generate, store and use energy.

\section{Acknowledgements}

This manuscript is based upon work supported by the startup funds provided by the College of Engineering at the University of Rhode Island.

\section{Notes and references}

1 H. Zhu, Y. Fu, F. Meng, X. Wu, Z. Gong, Q. Ding, M. V. Gustafsson, M. T. Trinh, S. Jin and X.-Y. Zhu, Nature Materials, 2015, 14, 636.

2 H. J. Snaith, The Journal of Physical Chemistry Letters, 2013, 4, 3623-3630.

3 Y. Fang, Q. Dong, Y. Shao, Y. Yuan and J. Huang, Nature Photonics, 2015, 9, 679.

4 M. Yuan, L. N. Quan, R. Comin, G. Walters, R. Sabatini, O. Voznyy, S. Hoogland, Y. Zhao, E. M. Beauregard, P. Kanjanaboos, Z. Lu, D. H. Kim and E. H. Sargent, Nature Nanotechnology, 2016, 11, 872.

5 B. Saparov and D. B. Mitzi, Chemical Reviews, 2016, 116, 4558-4596.

6 L. Dou, A. B. Wong, Y. Yu, M. Lai, N. Kornienko, S. W. Eaton, 
A. Fu, C. G. Bischak, J. Ma, T. Ding, N. S. Ginsberg, L.-W. Wang, A. P. Alivisatos and P. Yang, Science, 2015, 349, 15181521.

7 M. Saliba, T. Matsui, J.-Y. Seo, K. Domanski, J.-P. CorreaBaena, M. K. Nazeeruddin, S. M. Zakeeruddin, W. Tress, A. Abate, A. Hagfeldt and M. Grätzel, Energy Environ. Sci., 2016, 9, 1989-1997.

8 J. Burschka, N. Pellet, S.-J. Moon, R. Humphry-Baker, P. Gao, M. K. Nazeeruddin and M. Grätzel, Nature, 2013, 499, 316.

9 H. Tsai, W. Nie, J.-C. Blancon, C. C. Stoumpos, R. Asadpour, B. Harutyunyan, A. J. Neukirch, R. Verduzco, J. J. Crochet, S. Tretiak, L. Pedesseau, J. Even, M. A. Alam, G. Gupta, J. Lou, P. M. Ajayan, M. J. Bedzyk and M. G. Kanatzidis, Nature, 2016, 536, 312-316.

10 A. Z. Chen, M. Shiu, J. H. Ma, M. R. Alpert, D. Zhang, B. J. Foley, D.-M. Smilgies, S.-H. Lee and J. J. Choi, Nature Communications, 2018, 9, 1336.

11 A. Jaffe, Y. Lin and H. I. Karunadasa, ACS Energy Letters, 2017, 2, 1549-1555.

12 G. Liu, L. Kong, W. Yang and H. kwang Mao, Materials Today, 2019, 27, $91-106$.

13 L. Kong, G. Liu, J. Gong, Q. Hu, R. D. Schaller, P. Dera, D. Zhang, Z. Liu, W. Yang, K. Zhu, Y. Tang, C. Wang, S.-H. Wei, T. Xu and H.-k. Mao, Proceedings of the National Academy of Sciences, 2016, 113, 8910-8915.

14 M. Szafrański and A. Katrusiak, The Journal of Physical Chemistry Letters, 2017, 8, 2496-2506.

15 L. Zhang, C. Liu, L. Wang, C. Liu, K. Wang and B. Zou, Angewandte Chemie International Edition, 2018, 57, 11213-11217.

16 G. Liu, L. Kong, J. Gong, W. Yang, H.-k. Mao, Q. Hu, Z. Liu, R. D. Schaller, D. Zhang and T. Xu, Advanced Functional Materials, 2017, 27, 1604208.

17 A. Jaffe, Y. Lin, W. L. Mao and H. I. Karunadasa, Journal of the American Chemical Society, 2017, 139, 4330-4333.

18 A. Jaffe, Y. Lin, C. M. Beavers, J. Voss, W. L. Mao and H. I. Karunadasa, ACS Central Science, 2016, 2, 201-209.

19 G. A. Elbaz, W.-L. Ong, E. A. Doud, P. Kim, D. W. Paley, X. Roy and J. A. Malen, Nano Letters, 2017, 17, 5734-5739.

20 X. Qian, X. Gu and R. Yang, Applied Physics Letters, 2016, 108, 063902.

21 M. Wang and S. Lin, Advanced Functional Materials, 2016, 26, 5297-5306.

22 B. Li, Y. Kawakita, Y. Liu, M. Wang, M. Matsuura, K. Shibata, S. Ohira-Kawamura, T. Yamada, S. Lin, K. Nakajima and S. F.
Liu, Nature Communications, 2017, 8, 16086 EP -.

23 A. Pisoni, J. Jacimovic, O. S. Barisic, M. Spina, R. Gaal, L. Forro and E. Horvath, The Journal of Physical Chemistry Letters, 2014, 5, 2488-2492.

24 C. Caddeo, C. Melis, M. I. Saba, A. Filippetti, L. Colombo and A. Mattoni, Phys. Chem. Chem. Phys., 2016, 18, 24318 24324.

25 A. Giri, A. Z. Chen, A. Mattoni, K. Aryana, D. Zhang, X. Hu, S.-H. Lee, J. J. Choi and P. E. Hopkins, Nano Letters, 2020, 20, 3331-3337.

26 D. A. Dalton, W.-P. Hsieh, G. T. Hohensee, D. G. Cahill and A. F. Goncharov, Sci. Rep., 2013, 3, 2400 EP -.

27 B. Chen, W.-P. Hsieh, D. G. Cahill, D. R. Trinkle and J. Li, Phys. Rev. B, 2011, 83, 132301.

28 W.-P. Hsieh, M. D. Losego, P. V. Braun, S. Shenogin, P. Keblinski and D. G. Cahill, Phys. Rev. B, 2011, 83, 174205.

29 G. T. Hohensee, M. R. Fellinger, D. R. Trinkle and D. G. Cahill, Phys. Rev. B, 2015, 91, 205104.

30 A. Giri and P. E. Hopkins, Phys. Rev. B, 2017, 96, 220303.

31 G. T. Hohensee, R. B. Wilson and D. G. Cahill, Nat Commun, 2015, 6, year.

32 W.-P. Hsieh, A. S. Lyons, E. Pop, P. Keblinski and D. G. Cahill, Phys. Rev. B, 2011, 84, 184107.

33 A. Giri, J. T. Gaskins, L. Li, Y.-S. Wang, O. V. Prezhdo and P. E. Hopkins, Phys. Rev. B, 2019, 99, 165139.

34 G. Profeta, C. Franchini, N. N. Lathiotakis, A. Floris, A. Sanna, M. A. L. Marques, M. Lüders, S. Massidda, E. K. U. Gross and A. Continenza, Phys. Rev. Lett., 2006, 96, 047003.

35 A. Mattoni, A. Filippetti, M. I. Saba and P. Delugas, The Journal of Physical Chemistry C, 2015, 119, 17421-17428.

36 A. Mattoni, A. Filippetti, M. I. Saba, C. Caddeo and P. Delugas, The Journal of Physical Chemistry Letters, 2016, 7, 529-535.

37 F. Brivio, J. M. Frost, J. M. Skelton, A. J. Jackson, O. J. Weber, M. T. Weller, A. R. Goñi, A. M. A. Leguy, P. R. F. Barnes and A. Walsh, Phys. Rev. B, 2015, 92, 144308.

38 K. Sääskilahti, J. Oksanen, J. Tulkki and S. Volz, Phys. Rev. B, 2014, 90, 134312.

39 B. Ramos-Alvarado and S. Kumar, The Journal of Physical Chemistry C, 2017, 121, 11380-11389.

40 P. Postorino and L. Malavasi, The Journal of Physical Chemistry Letters, 2017, 8, 2613-2622.

41 T. M. Brenner, D. A. Egger, L. Kronik, G. Hodes and D. Cahen, Nature Reviews Materials, 2016, 1, 15007.

42 Y. Zhao and K. Zhu, Chem. Soc. Rev., 2016, 45, 655-689. 